\title{
Driving Rate Effects in Avalanche-Mediated First-Order Phase Transitions
}

\author{
Francisco-José Pérez-Reche, ${ }^{1}$ Bosiljka Tadić, ${ }^{2}$ Lluís Mañosa, ${ }^{1}$ Antoni Planes, ${ }^{1}$ and Eduard Vives ${ }^{1, *}$ \\ ${ }^{1}$ Departament d'Estructura i Constituents de la Matèria, Universitat de Barcelona, \\ Diagonal 647, Facultat de Física, 08028 Barcelona, Catalonia, Spain \\ ${ }^{2}$ Department for Theoretical Physics, Jozef Stefan Institute, P.O.Box 3000, SI-1001, Ljubljana, Slovenia
}

(Received 4 June 2004; published 3 November 2004)

\begin{abstract}
We study the driving-rate and temperature dependence of the power-law exponents that characterize the avalanche distribution in first-order phase transitions. Measurements of acoustic emission in structural transitions in $\mathrm{Cu}-\mathrm{Zn}-\mathrm{Al}$ and $\mathrm{Cu}-\mathrm{Al}-\mathrm{Ni}$ are presented. We show how the observed behavior emerges within a general framework of competing time scales of avalanche relaxation, driving rate, and thermal fluctuations. We confirm our findings by numerical simulations of a prototype model.
\end{abstract}

DOI: 10.1103/PhysRevLett.93.195701

PACS numbers: 64.60.My, 64.60.Qb, 81.30.Kf

Introduction.-The dynamics of first-order phase transitions (FOPT) in the presence of disorder is a longstanding problem that is undergoing renewed interest. Besides the phenomena of nucleation, metastability, and hysteresis, additional features are presently being revisited: (i) the extension of the transition over a broad region on the generic temperature-field phase diagram (the field is the intensive parameter conjugated to the order parameter); and (ii) the fact that transitions occur through a sequence of avalanches that link metastable states when thermal fluctuations are not dominant at low enough temperatures. These order parameter discontinuities are associated with the sudden transformation of a fraction of the system. This behavior is observed in many magnetic [1-4], superconducting [5,6], and structural $[7,8]$ transitions and in vapor condensation on porous media [9]. In many cases this phenomenon has been described as "athermal" FOPT [8]. In practice, this means that for the FOPT to proceed, it is necessary to drive the system externally by varying the temperature $T$ or the generic field $H$. When $T$ and $H$ are constant, no avalanches are detected even in the case of long waiting times. This reflects the fact that thermal fluctuations are irrelevant compared with the high-energy barriers separating the metastable states, and that temperature acts as a scalar field. The transformed fraction as a function of time only depends on the present and past values of $T$ or $H$, but not explicitly on time.

In different systems, avalanches have been detected with a variety of experimental techniques: induction (Barkhausen) [4,10], magnetization [11], calorimetry [12], resistivity [5], acoustic emission (AE) [7], capacitance [9], and optical measurements [1,3,13]. In many cases the statistical distribution of the avalanche properties (area, duration, etc.) exhibits power-law behavior over several decades. This has suggested the existence of criticality and has stimulated the search for universality in the power-law exponents. However, many different exponent values have been reported and comparison with the theoretically predicted universality classes [14-16] is controversial. Experimentally accessible quantities such as the distribution of signal amplitudes are not straightforwardly comparable with those obtained in numerical simulations such as the avalanche sizes. Moreover, the experimental distributions in some cases show exponential corrections that make it difficult to obtain the powerlaw exponents.

Systematic measurements with well-controlled external parameters are scarce. In structural transitions, the exponent associated with $\mathrm{AE}$ distributions has been shown to depend on repetitive cycling through the transition. Stationary values, which can be grouped into different classes, are obtained after a relatively large number of cycles [17]. For Barkhausen measurements in Fe films, an increase of the exponent has been found when temperature is decreased from room temperature to $10 \mathrm{~K}$ [18]. For soft-magnetic materials, two classes have been proposed [19]: one exhibiting exponents that decrease with increasing driving-rate $r$ and the other with exponents that are independent of $r$ [20].

In this Letter we present systematic measurements of the influence of cooling rate $r=|\dot{T}|$ on the distribution of amplitudes of AE signals in structural transitions. We analyze two systems with different degrees of athermal character. In one case, the exponent has been found to decrease with $r$, while it increases in the other case. Results are well explained within a general framework, which accounts for the effect of thermal fluctuations. Numerical simulations corroborate the proposed framework.

Experimental.-The studied single crystals [8] are $\mathrm{Cu}_{68.0} \mathrm{Zn}_{16.0} \mathrm{Al}_{16.0}$ and $\mathrm{Cu}_{68.4} \mathrm{Al}_{27.8} \mathrm{Ni}_{3.8}$, with martensitic transition temperatures $T_{M}=245 \mathrm{~K}$ and $T_{M}=257 \mathrm{~K}$, respectively. Both transitions extend in temperature $\Delta T \sim 35 \mathrm{~K}$. Previous results [8] confirmed that the $\mathrm{Cu}-$ $\mathrm{Zn}$-Al behaves athermally for temperature driving rates larger than a value that is less than $0.1 \mathrm{~K} / \mathrm{min}$. This was verified by checking that the AE activity per temperature interval as a function of $T$ is independent of $r$ (scaling). In contrast, $\mathrm{Cu}-\mathrm{Al}-\mathrm{Ni}$ is much less athermal. Although it 
clearly shows avalanche dynamics, the activity does not scale at the studied driving rates and few isothermal signals occur. It was estimated that scaling for this sample will hold for $r$ above $50 \mathrm{~K} / \mathrm{min}$.

The AE signals were detected by a piezo-electric transducer using the experimental setup explained elsewhere [8]. The samples were cycled through the transition more than 100 times in order to reach the stationary path. Individual AE signals were detected during cooling runs at selected rates and their amplitudes $A$ were analyzed. The experimentally accessible range is $4 \times$ $10^{-5} \mathrm{~V}<A<1.5 \times 10^{-3} \mathrm{~V}$. For the $\mathrm{Cu}$-Al-Ni sample averages, over $\sim 10$ cycles were also performed in order to obtain good statistics. The typical number of analyzed signals for each rate is $\sim 10^{5}$. The temperatures between which the cycles are performed are always the same: $T=$ $220 \mathrm{~K}$ and $T=320 \mathrm{~K}$ for the $\mathrm{Cu}-\mathrm{Zn}-\mathrm{Al}$ sample and $T=$ $220 \mathrm{~K}$ and $T=340 \mathrm{~K}$ for $\mathrm{Cu}-\mathrm{Al}-\mathrm{Ni}$. The distribution of AE amplitudes (as well as the size distribution in the simulations presented below) is studied by the maximum likelihood method, which is independent of the way histograms are plotted. We have fitted the data to the normalized probability law: $p(A) \sim A^{-\alpha} e^{-\lambda A}$. Two different fits are performed: first, a one-parameter fit of the exponent $\alpha$ by imposing $\lambda=0$ and second, a twoparameter fit of $\alpha$ and $\lambda$. The values of $\alpha$ reported here correspond to the first fitted value, but error bars include the second fitted value. For the second fit, $|\lambda|<1000 \mathrm{~V}^{-1}$ in all cases, which indicates that differences between the two fitted functions start to be important outside of the experimental window. Figure 1 shows the dependence of $\alpha$ on the rate $r$. The striking feature is that for the most athermal case ( $\mathrm{Cu}-\mathrm{Zn}-\mathrm{Al}), \alpha$ decreases with $r$ while for the less athermal sample $(\mathrm{Cu}-\mathrm{Al}-\mathrm{Ni})$, it increases. Moreover, in both cases, the dependence on $r$ is logarith-

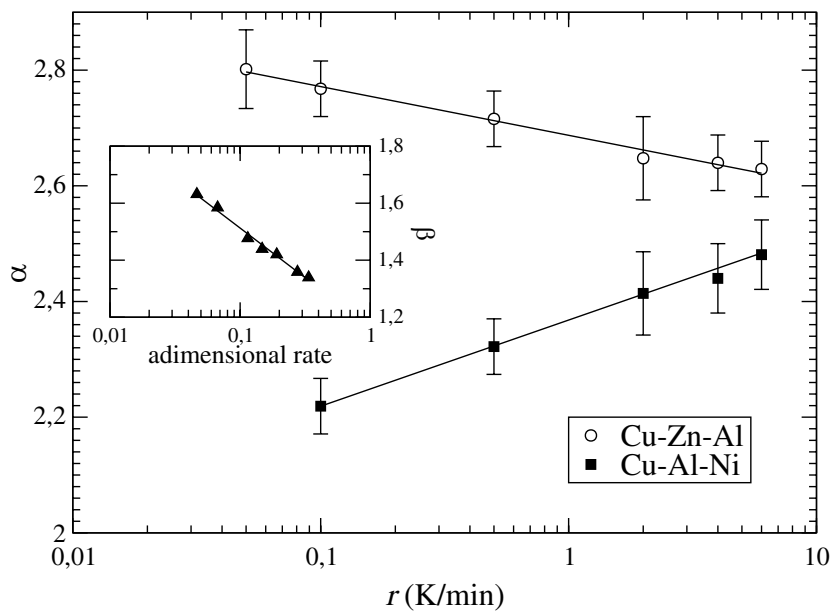

FIG. 1. Fitted exponents corresponding to the amplitude distribution of $\mathrm{AE}$ signals as a function of the drivingrate $r$. The inset shows the exponents corresponding to the Barkhausen signal integrated amplitudes from Ref. [20] mic, as shown by the fitted lines. A decrease of the exponent of Barkhausen avalanche sizes (area below the signals) in polycrystalline $\mathrm{Si}-\mathrm{Fe}$ with increasing rate is reported in Ref. [20]. We have plotted these data on a loglinear scale in the inset of Fig. 1, and a good logarithmic dependence is also observed.

Modeling.-Understanding of the results comes from the analysis of the relevant time scales involved in the problem. These are: (i) the time scale of the avalanche relaxation $\tau_{\mathrm{av}}$. Although avalanche durations are known to be also power-law distributed over several decades, the detected values range between $\tau_{\mathrm{av}} \sim 10^{-6}-10^{-3} \mathrm{~s}$. (ii) The characteristic time associated with the driving rate $\tau_{\mathrm{dr}}$. This can be conveniently defined as a function of the extension of the FOPT as $\tau_{\mathrm{dr}}=\Delta T / r$. In our case from the experimentally accessible rates, $\tau_{\mathrm{dr}} \sim 10^{2}-10^{4} \mathrm{~s}$. (iii) The third time scale is associated with the activation of the FOPT by thermal fluctuations $\tau_{\text {th }}$, which decreases with temperature. It can be estimated using the model in Ref. [8]. For $\mathrm{Cu}-\mathrm{Al}-\mathrm{Ni}, \tau_{\text {th }} \lesssim 10^{2} \mathrm{~s}$ is obtained (from the inverse of the fitted value of the characteristic frequency $\omega)$. For $\mathrm{Cu}-\mathrm{Zn}-\mathrm{Al}$, isothermal experiments indicate a lower bound $\tau_{\text {th }}>10^{5} \mathrm{~s}$. This difference is likely to be due to the different mechanisms (slipping for $\mathrm{Cu}-\mathrm{Zn}-\mathrm{Al}$ and twinning for $\mathrm{Cu}-\mathrm{Al}-\mathrm{Ni}$ ) for the transitions which exhibit quite a different rate of formation $\left(\tau_{\text {th }}^{\text {twin }} \ll \tau_{\text {th }}^{\text {slip }}\right)$ [21]. Moreover, given that $\Delta T / T_{M} \sim 0.1$ is small for the studied samples, we expect that, in both cases, $\tau_{\text {th }}$ does not depend on $r$.

The dynamics of the FOPT will therefore be determined by competition between these three time scales. In the "adiabatic" limit $\tau_{\mathrm{dr}} / \tau_{\mathrm{th}} \rightarrow 0$ and $\tau_{\mathrm{av}} / \tau_{\mathrm{dr}} \rightarrow 0$, i.e., when the time scales are well separated $\tau_{\mathrm{av}} \ll \tau_{\mathrm{dr}} \ll \tau_{\text {th }}$, the avalanches are well defined and both temperature and finite driving rate do not affect avalanche scaling. When $\tau_{\mathrm{dr}}$ approaches $\tau_{\mathrm{av}}$, the system will still display avalanche behavior but will start to overlap. Typically, small avalanches merge to form larger signals [22]. This leads to a decrease in the exponent $\alpha$ when $\tau_{\mathrm{av}} / \tau_{\mathrm{dr}} \propto r$ increases. On the other hand, by increasing temperature and or decreasing driving rate, competition of the thermal fluctuations and driving may produce diverse effects. Strictly speaking, for any nonzero value of the ratio $\tau_{\mathrm{dr}} / \tau_{\mathrm{th}} \propto 1 / r$, the activity may not stop even at $r=0$ until thermal equilibrium is reached, making the concept of avalanches difficult to define. Nevertheless, from the experimental point of view, there is always a threshold below which signals cannot be detected. Thus, distinct signals can still be measured for low enough values of $\tau_{\mathrm{dr}} / \tau_{\mathrm{th}}$. We expect that increasing thermal activation (decreasing $\tau_{\text {th }}$ ) will help the advance of the FOPT by promoting the transformation of domains which otherwise would not transform under athermal circumstances, thus resulting again in the merging of small avalanches. On the other hand, thermal fluctuations may initiate small avalanches when 
the system is slowly driven ( $\tau_{\mathrm{dr}}$ is large) which will not initiate at such early fields under fast driving. The potential effects of the two time scale ratios $\tau_{\mathrm{av}} / \tau_{\mathrm{dr}}$ and $\tau_{\mathrm{dr}} / \tau_{\text {th }}$ on the scaling exponents are shown schematically in Fig. 2. Generally, for a given system at finite temperature, driving-rate dependence is determined along the projected curve on the basal plane. Thus, depending on the experimentally accessible range of driving rates we can find an increase of the exponent for the less athermal samples, a region of constant exponents, or a decrease of the exponent for the more athermal samples. This enlarged space of parameters offers a suitable scenario to understand the present results for the AE measurements, as well as the behavior observed by large temperature changes recently reported [18]. The studied case of Barkhausen avalanches $[19,20]$ represents the limit where thermal fluctuations can be neglected.

In order to substantiate the above arguments, we have performed numerical simulations. The prototype model for hysteresis and avalanches in athermal FOPT is the 3DRandom Field Ising Model (RFIM) at $T=0$ with metastable dynamics [14]. It consists of a ferromagnetic Ising model with local random fields which are Gaussian distributed with standard deviation $\sigma$. The system is driven from the saturated state by decreasing the external field $H$. Spins flip according to a local relaxation rule, which is responsible for hysteresis. By construction, the model is in the perfect adiabatic limit $\tau_{\mathrm{av}} \ll \tau_{\mathrm{dr}} \ll \tau_{\text {th }}=\infty$. The first inequality is guaranteed because as soon as one avalanche starts, $H$ is kept constant until the system reaches a new metastable state. We will refer to such dynamics as athermal-adiabatic dynamics. The model reproduces the existence of avalanches whose size $s$ and duration $\Delta t$ can be statistically analyzed. In a large region close to the critical point $\sigma_{c} \simeq 2.21$ [23], the avalanche sizes corresponding to a full half-loop distribute, to a good approximation, according to a power law. Sensitivity of the exponent to system size $L$ and $\sigma$ has recently been studied in detail [23]. Moreover, given that

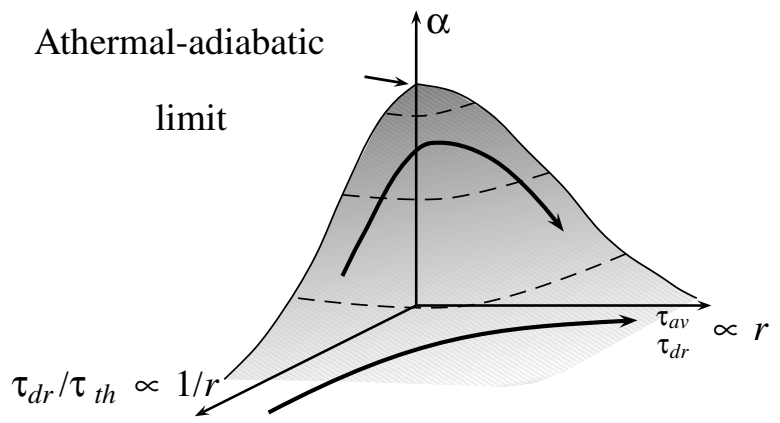

FIG. 2. Schematic diagram showing the behavior of the exponent as a function of the relevant time scales. The hyperbola on the base plane shows the behavior at constant temperature when increasing the driving rate. the distributions are only approximate power laws, the exponents also depend on the fitting range.

Extension of the model in order to incorporate the finite driving rate $r=|\dot{H}|$ and thermal fluctuations effects is not straightforward. The appropriate enlargement of the parameter space can be achieved in several ways. Here we adapt the approach in which the separation of signals is preserved to a large degree. A first attempt to incorporate the finite driving rate, which we refer to as athermal-step-driven dynamics, was proposed in Refs. $[24,25]$. (Here we extend this work to lower driving rates.) It consists of increasing the external field $H$ by a certain step $\Delta H$ and keeping it fixed until a new metastable situation is reached. The obtained signals are superpositions of the avalanches of the athermal-adiabatic model. The statistical analysis shows that the exponent decreases with the increasing "rate" (quantified by $\Delta H$ ). Results for a system with $\sigma=2.0$ are shown in Fig. 3 as filled circles [26]. Data are compared with the exponent corresponding to athermal-adiabatic dynamics, which is shown by a dashed horizontal line. A logarithmic decrease with $r$ is obtained in a broad region, in qualitative agreement with the results obtained for $\mathrm{Cu}-\mathrm{Zn}-\mathrm{Al}$ [27].

Strictly speaking, for the athermal-step-driven dynamics, $r$ is not constant along the hysteresis path. This could be important since long avalanches are known to concentrate on the central part of the hysteresis loop and correlations may exist. In order to check whether or not this affects the results, we propose other dynamics which we will call athermal-finite-driving-rate dynamics. Using the original athermal-adiabatic dynamics, one records the sequence of avalanches in half a loop (including the field value for which they occur, their size, and their duration). For a given finite rate $\dot{H}$, it is possible to analyze the sequence of recorded avalanches and determine which of them will overlap. Thus, one can obtain the

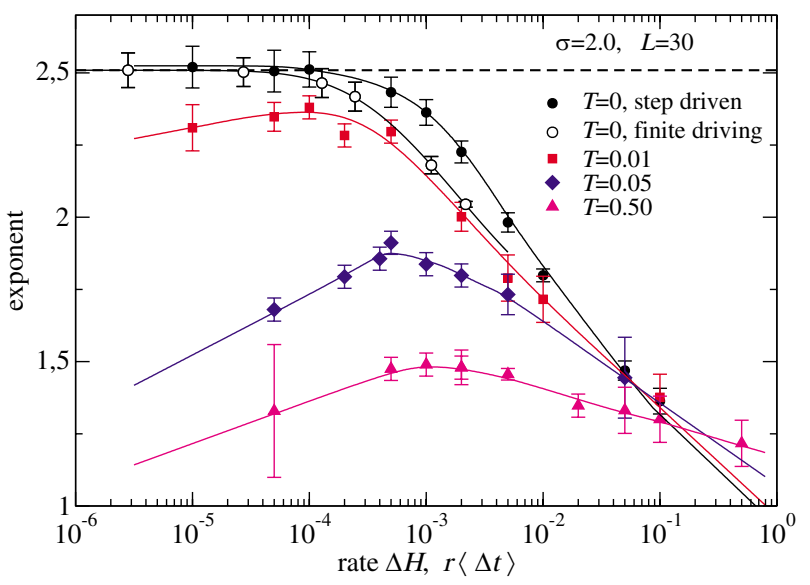

FIG. 3 (color online). Exponents corresponding to the avalanche size distribution as a function of the driving rate obtained by different numerical algorithms at different temperatures $T$, as explained in the text. 
modified sequence of signals and perform the statistical analysis. Results obtained by this method are indicated in Fig. 3 as open circles. In order to compare it with the previous data, $\dot{H}$ has been multiplied by the average duration $\langle\Delta t\rangle$ of the pulses in a half loop. Agreement with the previous dynamics is very good, thus supporting the use of step-driven dynamics in simulations.

The numerical results obtained are in agreement with the behavior proposed in Fig. 2 and correspond to the $\tau_{\mathrm{dr}} / \tau_{\mathrm{th}}=0$ plane. To simulate the effect of a finite $\tau_{\mathrm{th}}$, we must modify the dynamics proposed above introducing the effect of thermal fluctuations, but still keeping separate signals. This is achieved by slightly modifying the step-driven algorithm. After each $\Delta H$, not only unstable spins relax, but also an extra small fraction of locally stable spins is reversed towards the new phase due to thermal activation. This is done using the Metropolis algorithm, simultaneously and independently (parallel updating) for each unreversed spin, with a probability $p=\min \left(1, e^{-\Delta E / T}\right)$, where $\Delta E$ is the energy change associated with the reversal of each spin. During the subsequent evolution of the avalanche, the field is kept constant and temperature is set to 0 . The exponents for three different temperatures are shown in Fig. 3. As can be seen, nonmonotonous behavior is obtained, as qualitatively illustrated in Fig. 2.

Several models have been previously proposed for the understanding of the influence of driving rate on the power-law exponents characterizing signal distributions $[4,22,28,29]$. All of them correspond to athermal situations and account for the decrease of the exponent with increasing $r$, as a consequence of the overlap of small avalanches. In some cases a linear decrease of the exponents with $r$ is predicted. Our experiments and simulations fit better with a $\log r$ dependence. Moreover, we have also shown that Barkhausen data in Ref. [20] also conform to this logarithmic tendency. We expect that the origin of this functional dependence lies in the correlation between avalanches evolving in the restricted geometry of the transforming sample rather than in the dynamics itself. Such correlations have not been taken into account in previous models that correspond to single advancing interfaces in magnets $[4,28,29]$ or systems with uncorrelated avalanches [22].

Conclusion.-We have shown that the avalanches at FOPT may exhibit a variety of the driving-rate dependences related to the interplay between the three relevant time scales. We have, therefore, clarified under which experimental circumstances one can expect exponents similar to those predicted in the athermal-adiabatic limit.

We thank A. Saxena for fruitful comments. This work has received financial support from CICyT (Spain), Project No. MAT2004-01291 and CIRIT (Catalonia), Project No. 2001SGR00066. B. T. acknowledges support from DURSI (Catalonia) and Project No. P1-0044 of the Ministry of Education, Science, and Sports (Slovenia). F. J. P.-R. acknowledges support from DGICyT (Spain).

*Electronic address: eduard@ecm.ub.es

[1] K. L. Babcock and R. M. Westervelt, Phys. Rev. Lett. 64, 2168 (1990).

[2] P. J. Cote and L.V. Meisel, Phys. Rev. Lett. 67, 1334 (1991).

[3] E. Puppin, Phys. Rev. Lett. 84, 5415 (2000).

[4] G. Durin and S. Zapperi, cond-mat/0404512.

[5] W. Wu and P.W. Adams, Phys. Rev. Lett. 74, 610 (1995).

[6] E. Altshuler and T. H. Johansen, Rev. Mod. Phys. 76, 471 (2004), and references therein.

[7] E. Vives et al., Phys. Rev. Lett. 72, 1694 (1994).

[8] F. J. Pérez-Reche, E. Vives, Ll. Mañosa, and A. Planes, Phys. Rev. Lett. 87, 195701 (2001).

[9] M. P. Lilly, P.T. Finley, and R. B. Hallock, Phys. Rev. Lett. 71, 4186 (1993).

[10] H. Barkhausen, Phys. Z. 20, 401 (1919).

[11] V. Hardy et al., Phys. Rev. B 69, 020407(R) (2004).

[12] Ll. Carrillo and J. Ortín, Phys. Rev. B 56, 11508 (1997).

[13] D.-H. Kim, S. B. Choe, and S. C. Shin, Phys. Rev. Lett. 90, 087203 (2003).

[14] J. P. Sethna et al., Phys. Rev. Lett. 70, 3347 (1993).

[15] E. Vives, J. Goicoechea, J. Ortín, and A. Planes, Phys. Rev. E 52, R5 (1995).

[16] E. Vives and A. Planes, Phys. Rev. B 63, 134431 (2001).

[17] Ll. Carrillo et al., Phys. Rev. Lett. 81, 1889 (1998).

[18] E. Puppin and M. Zani, J. Phys.: Condens. Matter 16, 1183 (2004).

[19] G. Durin and S. Zapperi, Phys. Rev. Lett. 84, 4705 (2000).

[20] G. Bertotti, G. Durin, and A. Magni, J. Appl. Phys. 75, 5490 (1994).

[21] Z. Nishiyama, Martensitic Transformations (Academic Press, London, 1978).

[22] R. A. White and K. A. Dahmen, Phys. Rev. Lett. 91, 085702 (2003).

[23] F. J.Pérez-Reche and E. Vives, Phys. Rev. B 67, 134421 (2003); Phys. Rev. B (to be published).

[24] B. Tadić, Phys. Rev. Lett. 77, 3843 (1996).

[25] B. Tadić, Eur. Phys. J. B 28, 81 (2002).

[26] The actual values of the exponent obtained with the different numerical algorithms discussed in this work depend on $\sigma, L$, and the fitting range of $s$. We have verified that for $2.0<\sigma<2.4$ and $L>20$, the qualitative behavior for $2<s<1000$ is that shown in Fig. 3 .

[27] The values of the exponents are not comparable since the simulations correspond to avalanche sizes and the experimental data correspond to amplitudes of AE signals.

[28] B. Alessandro, C. Beatrice, G. Bertotti, and A. Montorsi, J. Appl. Phys. 68, 2901 (1990); ibid.68, 2908 (1990).

[29] S. L. A. de Queiroz and M. Bahiana, Phys. Rev. E 64, 066127 (2001). 